\title{
Efecto de la densidad de semillas en la germinación de tres especies del género Astrophytum (Cactaceae)
}

\section{Effect of seed density in the germination of three species of Astrophytum (Cactaceae)}

\author{
Gisela Muro-Pérez ${ }^{1,2}$, Enrique Jurado ${ }^{1^{*}}$, Joel Flores $^{3}$, Jaime Sánchez-Salas ${ }^{1,2}$ \\ ${ }^{1}$ Facultad de Ciencias Forestales, Universidad Autónoma de Nuevo León. Apartado postal 41. Carretera Nac. 11. km 145, CP \\ 67700, Linares, N.L. México. \\ ${ }^{2}$ Facultad de Ciencias Biológicas, Universidad Juárez del Estado de Durango. Av. Universidad s/n. Fracc. Filadelfia, C.P. \\ 35010, Gómez Palacio, Dgo. México. \\ ${ }^{3}$ Instituto Potosino de Investigación Científica y Tecnológica. A.C, División de Ciencias Ambientales. Camino a la Presa San \\ José 2055, Col. Lomas 4a Sección, C.P. 78216, San Luis Potosí, México. \\ *enrique-jurado@hotmail.com
}

\begin{abstract}
RESUMEN
Se colectaron frutos de poblaciones naturales de Astrophytum capricorne, A. myriostigma y A. ornatum en Coahuila, Durango e Hidalgo, respectivamente, con el objetivo de evaluar el efecto de la agrupación de semillas en la germinación, comparando tratamientos de 10 semillas agrupadas y 10 semillas no agrupadas (individuales). Las tres especies mostraron mayor porcentaje de germinación en semillas individuales. La germinación fue igual de rápida en semillas agrupadas e individuales para las tres especies. Estos resultados contrastan con el fenómeno de "rivalidad entre hermanos" que plantea la inhibición de la germinación en altas densidades como un mecanismo para evitar la competencia entre semillas hermanas con el fin de disminuir la competencia entre plántulas.
\end{abstract}

Palabras Clave: Dispersión agrupada de semillas, competencia entre plántulas.

\begin{abstract}
Fruits were obtained from wild populations of Astrophytum capricorne, A. myriostigma and A. ornatum in Coahuila, Durango and Hidalgo, respectively, in order to evaluate the effect of grouping on seed germination, comparing treatments of 10 individual seeds and 10 grouped seeds. All three species showed higher germination for individual seeds. Germination speed was not related to seed density. These results are in contrast with "sibling rivalry" hypothesis in which competition between sister seeds can inhibit germination to avoid competition due to a high density of seedlings.
\end{abstract}

KEYwords: Clump seed dispersal, seedling competition.

\section{INTRODUCCIÓN}

Existen factores ambientales como el agua, temperatura y luz que promueven la germinación de las semillas (Baskin \& Baskin 2001); sin embargo, otros factores como la edad de las semillas (Baskin \& Baskin 2001) y la dispersión también influyen en la germinación pues de ella depende que las semillas lleguen a un sitio seguro para germinar (Harper 1977). Se ha determinado que en las zonas áridas la mayoría de las semillas germinan muy cerca de la planta madre (Ellner \& Schmida 1981), pues ésta se encuentra en un sitio con condiciones adecuadas para el crecimiento. Para las especies cuyas semillas se dispersan juntas (clump dispersal) la rivalidad entre semillas podría disminuir o inhibir la germinación en altas densidades con el fin de reducir la competencia entre plántulas, lo cual se ha interpretado como un mecanismo de regulación poblacional (Cheplick 1992, 1993, McMurray et al. 1997, Murray 1998, Lortie \& Turkington 2002, Grundy et al. 2003, Dyer 2004, Flores \& Jurado 2009). Sin embargo, también se ha registrado un incremento en la germinación de semillas agrupadas en altas densidades, lo cual se ha relacionado con mayor probabilidad de supervivencia en por lo menos un individuo (Linhart 1976, Waite \& Hutchings 1978, McMurray et al. 1997). Así, la competencia entre plántulas podría justificarse al aumentar la probabilidad de que la 
planta establezca al menos un descendiente, pero genera un conflicto con las semillas que podrían tener un mecanismo para evitar germinar en condiciones de alta competencia. Se esperaría que la alta densidad de semillas inhiba la germinación de las mismas para evitar la competencia, a menos que: (i) la probabilidad de establecimiento sea tan baja que la planta madre requiera de un gran número de plántulas para poder establecer una con éxito, o bien (ii) que las semillas no estén adaptadas a germinar juntas, pues por sus mecanismos de dispersión se distribuyen separadas en el paisaje.

La forma de agrupamiento de las semillas depositadas en el suelo en campo depende de la forma en que son dispersadas; por ejemplo, los frutos carnosos de algunas especies son consumidos por vertebrados frugívoros, los cuales dispersan semillas viables al defecar o regurgitar semillas en grupos (Cortés-Figueira et al. 1994, Corlett 1998, Traveset 1998, Traveset et al. 2012). La dispersión de semillas por roedores (McMurray et al. 1997) y por hormigas (Parker \& Kelly 1989) también puede resultar en bancos de semillas agrupados. Las semillas de Astrophytum spp. presentan hidrocoria, es decir, tienen características para ser dispersadas por agua (Bregman 1988, SánchezSalas et al. 2012). Sin embargo; existe evidencia de que también son dispersadas por hormigas (Terry et al. 2012), por lo cual pueden presentar banco de semillas agrupado.

El género Astrophytum Lem. se distribuye en el desierto Chihuahuense, principalmente en el matorral micrófilorosetófilo (Rzedowski 1983). Este desierto es considerado centro de alta diversidad, por lo que es prioritario realizar investigación y conservación de sus recursos naturales (Hernández \& Bárcenas 1995). Astrophytum capricorne (Dietr.) Britton et Rose crece asociado a matorral micrófilo en los estados mexicanos de San Luis Potosí, Coahuila y Nuevo León; Astrophytum myriostigma Lem. ocurre en hábitats de matorral rosetófilo en San Luis Potosí, Coahuila, Durango, Nuevo León y Tamaulipas, y Astrophytum ornatum (DC.) Weber ex Britton \& Rose se distribuye en matorrales xerófilos de Hidalgo y Querétaro (Bravo-Hollis \& Sánchez-Mejorada 1991).

La germinación del género Astrophytum ha sido ampliamente documentada (De la Rosa-Ibarra \& García 1994, Arredondo-Gómez \& Camacho-Morfín 1995, RiojaParadela \& Romero-Méndez 2002, Sánchez-Salas et al. 2006, 2012, Flores et al. 2011); sin embargo, no existen estudios sobre el efecto que tiene la agrupación de sus semillas en la germinación. La hipótesis de rivalidad entre semillas hermanas sugiere que una vez germinada una semilla, ésta inhibiría la germinación de las semillas aledañas para disminuir la competencia (Cheplick 1992, 1993, Grundy et al. 2003, Dyer 2004, Flores \& Jurado 2009). Así, aquí se evaluó el efecto de la agrupación de semillas en la germinación de Astrophytum capricorne, A. myriostigma y A. ornatum, tres especies endémicas amenazadas de extinción según la Norma Oficial Mexicana (Semarnat 2010). La hipótesis planteada es que las semillas en contacto cercano tendrían un menor porcentaje de germinación que las semillas separadas.

\section{MATERIALES Y MÉTODOS}

\section{ESPECIES DE ESTUDIO}

Se trabajó con tres especies del género Astrophytum: A. capricorne es de tallo subgloboso de $25 \mathrm{~cm}$ de altura, tiene de 7 a 8 costillas, de 6 hasta 20 espinas, la flor es de $6 \mathrm{~cm} \mathrm{de}$ longitud. Sus semillas son de $2.5 \mathrm{~mm}$ de espesor y brillantes (Bravo-Hollis \& Sánchez-Mejorada 1991). Astrophytum myriostigma es de tallo simple, tiene de 3 a 8 costillas, las aréolas son próximas, distantes entre sí de 8 a $15 \mathrm{~mm}$, no presenta espinas, la flor es campanuliforme, de $6 \mathrm{~cm}$ de longitud, de color amarillo, con semillas naviculares (BravoHollis \& Sánchez-Mejorada 1991). Astrophytum ornatum es de tallo esférico, de $35 \mathrm{~cm}$ de altura, con 8 costillas, rectas, y de 6 a 10 espinas radiales, presenta una espina central, raras veces 2, las flores crecen cercanas al ápice, de $9 \mathrm{~cm}$ de longitud, las semillas son de $3 \mathrm{~mm}$ de longitud (BravoHollis \& Sánchez-Mejorada 1991).

\section{RECOLECCIÓN DE SEMILLAS}

Las semillas para este estudio se obtuvieron de poblaciones naturales de los estados de Durango, Coahuila e Hidalgo, México, en octubre de 2008; en matorrales micrófilo y rosetófilo (Rzedowski 1983). Los lotes de semillas se mantuvieron a temperatura ambiente en bolsas de papel para evitar acumulación de humedad (Moreno et al. 1992), favoreciendo la maduración (Flores \& Jurado 2009) durante cinco meses. Las semillas no tuvieron tratamiento pregerminativo, ya que se pretendía evaluar el efecto que produce agrupar y no agrupar las semillas en la germinación sin promotores de la misma. Se seleccionaron con un estereoscopio semillas que no tuvieran malformaciones o fracturas.

\section{DisEÑO EXPERIMENTAL}

Para realizar este experimento se utilizaron 2 tratamientos de densidad de semillas. Las semillas fueron colocadas en cajas Petri, usando agar bacteriológico como sustrato. Se usaron un total de 50 semillas por tratamiento, con 10 semillas por caja. Las semillas agrupadas se pusieron juntas de tal manera que estuvieran en contacto directo unas con otras en el centro de la Caja Petri, mientras que las individuales se distribuyeron de manera uniforme con un espacio de $2 \mathrm{~cm}$ entre cada una. Las 5 cajas (consideradas como 5 repeticiones) por especie, utilizadas para cada tratamiento, fueron puestas en una cámara de germinación con un fotoperiodo de $12 \mathrm{~h}$ luz/12 h oscuridad, bajo una temperatura constante de $25^{\circ} \mathrm{C}$. 
Se llevó el registro diario de la germinación de las semillas durante 30 días, determinándose la germinabilidad al final del periodo, la cual se expresó como el porcentaje de semillas que germinaron pasados 30 días, así como la velocidad de germinación $\left(\mathrm{t}_{50}\right)$ calculada como el número de días transcurridos hasta obtener la mitad de las semillas que germinaron en 30 días (Flores \& Briones 2001).

\section{ANÁLISIS ESTADÍSTICOS}

Para cada variable de respuesta (porcentaje de germinación final y velocidad de germinación) se realizó un ANOVA bifactorial, siendo los factores la especie (tres niveles: $A$. myriostigma, A. capricorne y A. ornatum) y la densidad de semillas (juntas y separadas). Los datos de porcentaje de germinación fueron transformados mediante el arcoseno de la raíz cuadrada (Sokal \& Rohlf 1995) para cumplir con el requisito de distribución normal previo al análisis de varianza. Donde hubo diferencia entre medias está se detectó con una prueba de Tukey.

\section{RESULTADOS}

De acuerdo con la hipótesis planteada, para las tres especies la germinación fue mayor en semillas individuales que en agrupadas ( $\mathrm{F}=15,87, \mathrm{P}=0,0004$; Fig. 1). Sin embargo, hubo diferencias entre especies, A. capricorne (72\% agrupadas, $52 \%$ separadas) presentó mayor germinación que $A$. myriostigma (44\% agrupadas, $22 \%$ separadas) y ésta, a su vez, germinó más que $A$. ornatum ( $8 \%$ agrupadas, $2 \%$ separadas).

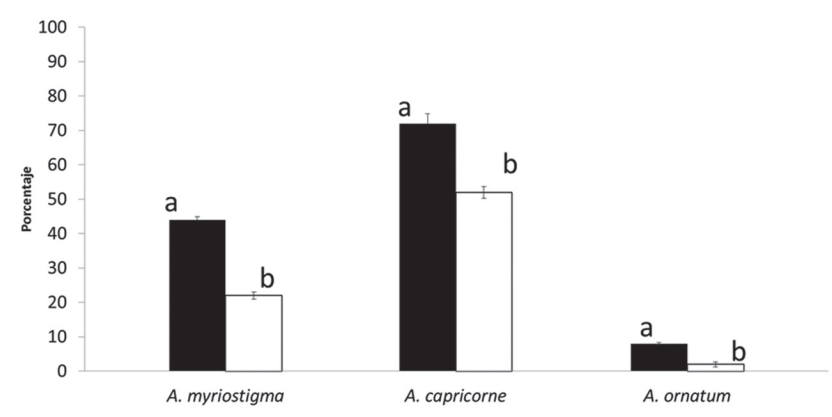

Figura 1. Porcentaje de germinación de semillas individuales (negro) y agrupadas (blanco) de tres especies del género Astrophytum. Cada porcentaje representa la media de cinco repeticiones con 10 semillas en cada una. Las barras de error corresponden al intervalo de confianza y las letras minúsculas diferentes indican diferencias entre las medias para cada especie (prueba de Tukey, $\mathrm{P} \leq 0,05$ ).

FIGURE 1. Germination percentage of individual seeds (black) and grouped seeds (white) from three species of Astrophytum. Each percentage is the mean of five replicates with 10 seeds in each one. Error bars are confidence intervals and different lower case letters indicate differences for each species (Tukey test, $\mathrm{P} \leq 0.05$ ).
No se encontraron diferencias en la velocidad de germinación entre semillas agrupadas e individuales, ni entre las especies ( $\mathrm{F}=0,394, \mathrm{P}=0,565$; Fig. 2).

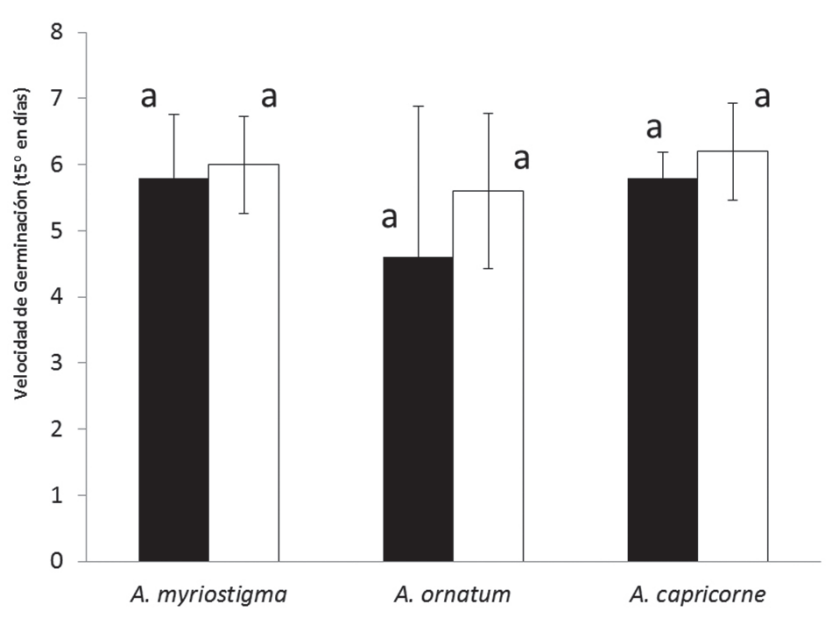

Figura 2. Comparación de la velocidad de germinación de las semillas individuales (negro) y agrupadas (blanco) de tres especies del género Astrophytum. Las barras de error muestran el intervalo de confianza (prueba de Tukey, $\mathrm{P} \leq 0,05$ ). Las letras minúsculas diferentes indican diferencias entre las medias para cada especie.

FIGURE 2. Comparison of germination rate between individual seeds (black) and grouped seeds (white) from three Astrophytum species. Error bars are confidence intervals and different lower case letters indicate differences for each species (Tukey test, $\mathrm{P} \leq$ $0.05)$.

\section{DISCUSIÓN}

En las tres especies de Astrophytum, la germinación fue mayor en semillas individuales que en agrupadas, lo cual coincide con la hipótesis de competencia por recursos y rivalidad de plántulas entre semillas hermanas (fenómeno de "sibling rivalry") que plantea una inhibición en la germinación en altas densidades con el fin de disminuir la competencia entre plántulas (Cheplick 1992, 1993, Grundy et al. 2003, Dyer 2004, Flores \& Jurado 2009). Estos resultados contrastan la expectativa de una mayor probabilidad de éxito de establecimiento de al menos una plántula (Linhart 1976, Waite \& Hutchings 1978, McMurray et al. 1997). Sin embargo, es importante destacar que si bien germinaron menos semillas agrupadas que individuales, hubo germinación en ambos casos. La supervivencia de plántulas dependientes de la densidad puede variar entre estaciones y entre especies (Lin et al. 2012). Además, la agrupación de semillas puede incrementar la probabilidad del establecimiento de plántulas bajo depredación de semillas por insectos y ataque por patógenos (Beckman et al. 2012). Estudios posteriores podrían explorar la calidad y sobrevivencia de las plántulas en función de su origen de 
germinación de semillas individuales o agrupadas.

En las especies de la familia Cactaceae es frecuente la producción masiva de semillas (Seal et al. 2009) y esta característica aumenta la probabilidad de que muchas semillas se encuentren juntas al germinar. Por otro lado, Sánchez-Salas et al. (2006) encontraron valores altos de germinación para Astrophytum myriostigma (cerca del $75 \%$ ) en semillas que se pusieron a germinar sin algún tipo de tratamiento que promoviera la germinación; aunque también evaluaron la germinación en semillas de diferente tamaño como semillas pequeñas y grandes. En ese estudio las semillas pequeñas obtuvieron germinación de $77 \%$; sin embargo no se evaluó si ésta era mayor en semillas agrupadas. En el presente estudio, las semillas de A. myriostigma agrupadas mostraron mayor germinación (44\%) que las semillas individuales $(22 \%)$, pero la germinación fue menor que la encontrada por Sánchez-Salas et al. (2006). Astrophytum ornatum presentó valores por debajo del 10\% de germinación en semillas agrupadas y $2 \%$ en semillas individuales. La baja germinación puede deberse a diferencias en sustratos, luz o fases de latencia en que se encuentren las semillas (Baskin \& Baskin 2001, Jurado \& Flores 2005). Astrophytum capricorne presentó valores más altos de germinación (72\%) en semillas individuales en comparación con $52 \%$ de germinación en semillas agrupadas. De manera similar, Isolatocereus dumortieri (Scheidw.) Backeb tuvo menor germinación con una mayor densidad de sus semillas (Flores \& Jurado 2009), sugiriendo una estrategia de germinación de semillas agrupadas con el fin de disminuir la competencia entre plántulas (Cheplick 1992, 1993, Grundy et al. 2003, Dyer 2004).

El tiempo $\left(\mathrm{t}_{50}\right)$ de germinación de las semillas de Astrophytum capricorne, A. myriostigma y A. ornatum fue 6 días. Estos resultados concuerdan con los obtenidos para otras cactáceas como Neobuxbaumia tetetzo (2-3 días; Flores \& Briones 2001), Turbinicarpus schmiedickeanus subsp. klinkerianus y T. schmiedickeanus subsp. macrochele (5 días, Flores et al. 2006) y Ferocactus histrix (4 días, PérezSánchez et al. 2011). También coinciden con los obtenidos para Stenocereus beneckei y Astrophytum myriostigma, cuyas semillas, dependiendo de su peso y edad, germinan en un período de 5 a 7 días (Ayala-Cordero et al. 2004, Sánchez-Salas et al. 2006). Así la germinación en ambientes áridos es rápida como lo propuesto por Jurado y Westoby (1992) por la corta duración de la humedad en el suelo.

\section{CONCLUSIONES}

Las semillas individuales de Astrophytum capricorne, $A$. myriostigma y $A$. ornatum presentaron mayores porcentajes de germinación que en las semillas agrupadas, tal como lo esperado con la hipótesis de rivalidad de plántulas. La velocidad de germinación de las semillas fue rápida y similar entre semillas individuales y agrupadas para cada especie.

\section{AGRADECIMIENTOS}

Consejo Nacional de Ciencia y Tecnología - Fondos Sectoriales - Secretaría de Educación Pública (2010-01156205). PAICYT- UANL.

\section{LITERATURA CITADA}

Arredondo-Gómez, A. \& F. Camacho-Morfín. 1995. Germinación de Astrophytum myriostigma (Lemaire) en relación con la procedencia de las semillas y temperatura de incubación. Cactáceas y Suculentas Mexicanas 40: 34-38.

Ayala-Cordero G., T. Terrazas, L. López-Mata \& C. Trejo. 2004. Variación en el tamaño y peso de la semilla y su relación con la germinación en una población de Stenocereus beneckei. Interciencia 29: 692-697.

BAskin, C.C. \& J.M. BAskin. 2001. Seeds: Ecology, Biogeography, and Evolution of Dormancy and Germination, pp. 293-329. Academic Press, San Diego. 666 pp.

Beckman, N.G., C. Neuhauser \& H.C. Muller-Landau. 2012. The interacting effects of clumped seed dispersal and distanceand density-dependent mortality on seedling recruitment patterns. Journal of Ecology 100: 862-873.

Bravo-Hollis, H. \& H. SÁnchez-Mejorada. 1991. Las Cactáceas de México, Vol. II. Universidad Nacional Autónoma de México, México, D.F. 404 pp.

Bregman, R. 1988. Forms of seed dispersal in Cactaceae. Acta Botanica Neerlandica 37: 395-402.

ChePlick, G.P. 1992. Sibling competition in plants. Journal of Ecology 80: 567-575.

Cheplick, G.P. 1993. Sibling competition is a consequence of restricted dispersal in an annual cleistogamous grass. Ecology 74: 2161-2164.

CoRlett, R.T. 1998. Frugivory and seed dispersal by vertebrates in the Oriental (Indomalayan) region. Biological Reviews of the Cambridge Philosophical Society 73: 413-448.

Cortés-Figueira, J.E., J. Vasconcellos-Neto, M.A. García \& A.L. TeixeIra de SouzA. 1994. Saurocory in Melocactus violaceus (Cactaceae). Biotropica 26: 295-301.

De la Rosa-Ibarra, M. \& H. García. 1994. Estimulación de la germinación de cinco especies de cactáceas consideradas en peligro de extinción. Phyton-International Journal of Experimental Botany 56: 147-150.

DYER, A.R. 2004. Maternal and sibling factors induce dormancy in dimorphic seed pairs of Aegilops triuncialis. Plant Ecology 172: 211-218.

Ellner, S. \& A. Schmida. 1981. Why are adaptations for longrange seed dispersal rare in desert plants? Oecologia 51:133144.

Flores, J. \& O. Briones. 2001. Plant life-form and germination in a Mexican inter-tropical desert: effects of soil water potential and temperature. Journal of Arid Environments 47: 485-497.

Flores, J. \& E. Jurado. 2009. Efecto de la densidad de semillas en la germinación de Isolatocereus dumortieri y Myrtillocactus 
geometrizans, cactáceas columnares endémicas de México. Revista Mexicana de Biodiversidad 80: 141-144.

Flores, J., E. Jurado \& A. Arredondo. 2006. Effect of light on germination of seeds of Cactaceae from the Chihuahuan Desert, México. Seed Science Research 16: 149-155.

Flores, J., E. Jurado, L. Chapa-Vargas, A. Ceroni-Stuva, P. Dávila-Aranda, G. Galíndez, D. Gurvich, P. León-Lobos, C. Ordóñes, P. Ortega Baes, N. Ramírez-Bullon, A. Sandoval, C.E. Seal, T. Ullian, \& H. W. Pritchard. 2011. Seeds photoblastism and its relationship with some plant traits in 136 cacti taxa. Enviromental and Experimental Botany 71: 79-88.

Grundy, A.C., A. Mead \& S. Burston. 2003. Modeling the emergence response of weed seeds to burial depth: interactions with seed density, weight and shape. Journal of Applied Ecology 40: 757-770.

HARPER, J.L. 1977. The population biology of plants. NY. Academic. 892 pp.

HernándeZ, H.M. \& R.T. BÁRCENAS. 1995. Endangered cacti in the Chihuahuan Desert: I. Distribution patterns. Conservation Biology 9: 1176-1188.

Jurado, E. \& M. Westoby. 1992. Germination biology of selected Central Australian plants. Australian Journal of Ecology 17: 341-348.

Jurado, E. \& J. Flores. 2005. Is seed dormancy under environmental control or bound to plant traits? Journal of Vegetation Sciences 16: 559-564.

Lin L., L.S. Comita, Z. Zheng \& M. CaO. 2012. Seasonal differentiation in density-dependent seedling survival in a tropical rain forest. Journal of Ecology 100: 905-914.

LinHART, Y.B. 1976. Density dependent seed germination strategies in colonizing versus non-colonizing plant species. Journal of Ecology 64: 375-380.

Lortie, C.J. \& R. Turkington. 2002. The effect of initial seed density on the structure of a desert annual plant community. Journal of Ecology 90: 435-445.

McMurray, M.H., S.H. Jenkins \& W.S. Longland. 1997. Effects of seed density on germination and establishment of a native and an introduced grass species dispersed by granivorous rodents. American Midland Naturalist 138: 322-330.

Moreno, N., J.J. López \& L. Arce. 1992. Aspectos sobre la germinación de Echinomastus mariposensis. Hester. Cactáceas y Suculentas Mexicanas 37: 21-27.

Murray, B.R. 1998. Density-dependent germination and the role of seed leachate. Australian Journal of Ecology 23: 411-418.

Parker, V.T. \& V.R. Kelly. 1989. Seed banks in California chaparral and other Mediterranean climate shrublands. In: M.A. Leek, V.T Parker \& R.L. Simpson (eds.), Ecology of Soil Seed Banks, pp. 231-255. Academic Press, California.

Pérez-Sánchez, R.M, E. Jurado, L. Chapa-Vargas \& J. Flores. 2011. Seed germination of Southern Chihuahuan Desert plants in response to elevated temperatures. Journal of Arid Environments 75: 978-980.

Rioja-Paradela, T.M. \& U. Romero-Méndez. 2002. Efecto del remojo sobre el potencial reproductivo sexual de Astrophytum myriostigma Lem. (Cactaceae) en condiciones controladas. Nakari 13: 21-34.

Rzedowski, J. 1983. Vegetación de México. Limusa, México, 478 pp.

SÁnchez-Salas, J., J. Flores \& E. Martínez-García. 2006. Efecto del tamaño de semilla en la germinación de Astrophytum myriostigma Lemaire. (Cactaceae), especie amenazada de extinción. Interciencia 31: 371-375.

Sánchez-Salas, J., E. Jurado, J. Flores, E. Estrada-Castillón \& G. Muro-PÉrEz. 2012. Desert species adapted for dispersal and germination during floods. Experimental evidence in two Astrophytum species (Cactaceae). Flora, in press. 207:707-711.

Seal, C.E., J. Flores, A. Ceroni-Stuva, P. Dávila-Aranda, P. León-Lobos, P. Ortega-Baes, G. Galíndez, M.A. AparicioGonzález, V. Castro-Cerero, M.I. Daws, M. Eason, C.M. Flores-Ortiz, P.A. del Fueyo, P. Olwell, C. Ordoñez, I. Peñalosa-Castro, R. Quintanar-Zúñiga, N. RamírezBullón, M. Rojas-Aréchiga, M. Rosas, A. Sandoval, W. Stuppy, T. Ulian, J. Vázquez-Medrano, H. Walter, M. Way \& H.W. Pritchard. 2009. The Cactus Seed Biology Database. Release 1. Royal Botanic Gardens, Kew.

SEMARNAT. 2010. Norma Oficial Mexicana NOM-059SEMARNAT-2010, Protección ambiental-Especies nativas de México de flora y fauna silvestres-Categorías de riesgo y especificaciones para su inclusión, exclusión o cambioLista de especies en riesgo. Secretaría de Medio Ambiente y Recursos Naturales. Diario Oficial de la Federación. México, D.F.

SoKAL, R.R. \& F.J. RohlF. 1995. Biometry: the principles and practice of statistics in biological research. Third edition. W.H. Freeman and Company, New York, USA. 887 pp.

Terry, M.K., A.E. Pepper, A.W. Strong, D.M. Tarin, D.M. Price \& J.R. MANHART. 2012. Genetic structure of a population of the endangered star cactus (Astrophytum asterias) in southern Texas. Southwestern Naturalist 57: 182-188.

Traveset, A. 1998. Effect of seed passage through vertebrate frugivores guts on germination: a review. Perspectives in Plant Ecology, Evolution and Systematics 1/2: 151-190.

Traveset, A., J.P. GonzÁlez-Varo \& A. Valido. 2012. Long-term demographic consequences of a seed dispersal disruption. Proceeding of the Royal Society B. 279:3298-3303. doi:10.1098/rspb.2012.0535

Waite, S. \& M.J. Hutchings. 1978. The effects of sowing density, salinity and substrate upon the germination of seeds of Plantago coronopus L. New Phytologist 81: 341-348.

Recibido: 09.11.12

Aceptado: 22.03.13 